\title{
Cloning of cDNAs for Two $\beta$-tubulin Isotypes Expressed in Murine T Cell Lymphoma, L5178Y and Analysis of Their Translation Products
}

\author{
Fumio Matsuzaki, Fumiko Harada, Yo-ichi Nabeshima* Yoshiaki Fujii- \\ Kuriyama* and Ichiro Yahara \\ Department of Cell Biology, The Tokyo Metropolitan Institute of Medical \\ Science, Honkomagome, Bunkyo-ku, Tokyo 113 and *Department of \\ Biochemistry, Cancer Institute, Japanese Foundation for Cancer Research, \\ Kami-Ikebukuro, Toshima-ku, Tokyo 170, Japan
}

\begin{abstract}
Three $\beta$-tubulin isoforms, $\mathbf{M} \beta \mathrm{Ib}, \mathbf{M} \beta \mathrm{Ia}$ and $\mathbf{M} \beta \mathrm{II}$, were detected by isoelectric focusing gel electrophoresis (IEF) in extracts of cultured cells of a mouse $\mathrm{T}$ cell lymphoma, L5178Y. To investigate the origin(s) of the isoforms, we have isolated cDNA clones encoding $\beta$-tubulin from a cDNA library prepared from poly (A) ${ }^{+}$RNA of L5178Y cells. Seventeen cDNA clones carrying the entire coding sequences of $\beta$-tubulin were isolated and classified into two distinct isotypes, represented by two clones designated pMT27 and pMT49, according to the results of restriction mapping. On the basis of the nucleotide sequences of the two cDNAs, pMT27 and pMT49 were identified as mouse $\beta$-tubulin isotypes 3 and 5 , respectively. By using in vitro translation products of hybrid-selected mRNAs and of the SP6 in vitro transcripts of the cDNAs, polypeptides encoded by the two cDNA clones were analyzed by IEF. We found that pMT27 and pMT 49 encode M $\beta$ Ib and M $\beta I$ Ia, respectively. In addition, $\mathrm{M} \beta \mathrm{II}$ was detected in translation products of mRNA specifically hybridized to pMT49, but not in those of the in vitro transcript of pMT49 DNA. These results suggest that $\mathrm{M} \beta \mathrm{II}$ is the translation product of mRNA whose $3^{\prime}$-untranslated region is highly homologous to that of pMT49.
\end{abstract}

Microtubules are a ubiquitous structure of eukaryotic cells, are composed of the heterodimer of $\alpha$ and $\beta$-tubulin subunits together with several species of associated proteins called MAPs, and function in various cellular events including mitosis, intracellular transport, morphogenesis, and motility (6). Recent progress in the biochemistry of microtubules has revealed the occurence of multiple tubulin isoforms, which are distinct from each other in IEF, in a single species and sometimes even in a single cell $(5,8,11)$. These isoforms are differentially expressed in tissues and during development $(14,19,23)$, raising the possibility that the tubulin isoform composition might be a factor which defines the multiple functions of microtubules $(4,9)$. Previous results show that the isoformal variations of tubulin are caused by differential expression of multiple tubulin genes $(2,14,19,23)$ and also by posttranslational modification or processing $(3,10)$. We recently found that cultured 
cells of L5178Y, a murine lymphoma cell line, express three major isoforms of $\beta$-tubulin, while normal $\mathrm{T}$ cell clones and other $\mathrm{T}$ lymphoma cell lines express only two of them (13). The current study was designed to investigate the origin of the three $\beta$-tubulin isoforms expressed in L5178Y cells.

\section{MATERIALS AND METHODS}

Materials. Avian myeloblastosis virus reverse transcriptase was purchased from Life Science Co.; the Klenow fragment of DNA polymerase and terminal deoxyribonucleotidyl transferase from Bethesda Research Laboratories; exonuclease Bal 31 from New England Biolabs; polynucleotide kinase from Boeringer Mannheim; restriction enzymes from Takara Shuzo Co., New England Biolabs, and Bethesda Research Laboratories; $\left[\alpha-{ }^{3}{ }^{2} \mathrm{P}\right] \mathrm{dCTP}$ $(5,000 \mathrm{Ci} / \mathrm{mmol}),\left[\alpha^{-32} \mathrm{P}\right] \mathrm{ATP}(5,000 \mathrm{Ci} / \mathrm{mmol})$, and $\left[{ }^{35} \mathrm{~S}\right] \mathrm{methionine}(>1000 \mathrm{Ci} / \mathrm{mmol})$ from Amersham.

Cloning procedure. Poly (A)+ RNA was prepared from L5178Y cells as described $(1,7)$. Double-stranded cDNA was synthesized and inserted into the vector pBR322 at the Pst I site by the GC-tailing method (20) to transform Echerichia coli HB101. Tetracyclineresistant transformants were screened by in situ colony hybridization (12) with the insert of chicken $\beta$-tubulin cDNA, pT2, as a probe (29). A cDNA clone encoding a partial sequence of mouse $\beta$-tubulin was initially identified by sequencing as described below. By using the insert of the mouse $\beta$-tubulin cDNA thus obtained, a new cDNA library was screened to isolate full length cDNA clones for $\beta$-tubulin. The second cDNA library was synthesized by the method of Land et al. (17) and fractionated by sucrose density gradient centrifugation. The first strand of the cDNA was tailed by oligo-TMP in place of oligo-dCMP for priming of the second strand of the cDNA (17).

DNA Sequence Analysis. The nucleotide sequences of the cDNA inserts were determined by the M13/dideoxy chain terminator method (27). Fragments for insertion into the polylinker site of the M13 vector mp18 or mp19 (24) were generated by direct digestion of the recombinant plasmids with appropriate restriction enzymes. In some experiments, recombinant plasmids were digested with restriction enzymes and trimmed by digestion with exonuclease Bal31, followed by repairing the ends of the DNA fragments with the Klenow fragment of $E$. coli DNA polymerase I and/or the second restriction digestion. The inserts of cDNA clones were sequenced in the two directions except for the last $75 \mathrm{bp}$ of the 3'-untranslated region of pMT49. A chemical degradation method (21) was used for sequencing of this region.

Construction of cDNA-specific probes. The 3'-untranslated sequences of the two cDNA clones pMT27 and pMT49 have no homology with each other (data not shown, see ref. 30), and were used for selective hybridization of specific mRNAs. cDNA inserts were digested with Bal 31 to remove their poly dA-dT and poly dG-dC tails and subcloned into the SmaI site of the replicative form of M13 mp18, followed by sequencing. BamHI cuts such recombinant DNAs derived from both pMT27 and pMT49 at one site approximately 300 base upstream from the stop codon, and at another site in the polylinker, producing short fragments containing $3^{\prime}$-untranslated regions of cDNAs. The BamHI fragment of a subclone of pMT27 was cleaved with $A l u$ at a site 2 bases downstream of the stop codon, and the $B a m \mathrm{HI}$ fragment of a subclone of pMT49 was cleaved with $S t u \mathrm{I}$ at a site 2 bases upstream of the stop codon. By this procedure, we constructed M13-UT27 containing 144 bases of the 3'-untranslated sequence of pMT27. Similarly, M13-UT49, which contained 105 bases of the $3^{\prime}$-untranslated sequence, was constructed from pMT49. 
Hybrid-selection of mRNAs. Double-stranded DNAs of M13-UT27 and M13-UT49 $(50 \mu \mathrm{g})$ were linearized by digestion with $E c o$ RI. By using the digests as a probe for each clone, specific mRNAs were selected from $35 \mu \mathrm{g}$ of total poly (A) ${ }^{+}$RNA as described (26). Nitrocellulose filters with DNA-RNA hybrid were washed with $10 \mathrm{mM}$ Tris- $\mathrm{HCl}(\mathrm{pH} 7.6)$, $1 \mathrm{mM}$ EDTA and $0.5 \% \mathrm{SDS}$ in the presence of $50 \mathrm{mM}$ or $150 \mathrm{mM} \mathrm{NaCl}$ at $65^{\circ} \mathrm{C}$; the RNAs were then eluted from the filters with distilled water at $100^{\circ} \mathrm{C}$.

In vitro transcription of cloned cDNAs. Plasmids containing $\beta$-tubulin sequence, pMT27 and pMT49, were digested with DraI to yield a fragment containing the entire cDNA insert. The fragment was then trimmed with Bal31 to remove poly dA-dT and poly dG-dC tails at the $5^{\prime}$-end of the cDNA inserts. The fragments were inserted into the M13 replicative form at the HincII site of the polylinker and sequenced from the two ends of the fragments to find fragments containing both initiation and termination codons. Among these clones, M13-MT27 had an insert containing the cDNA sequence from $47 \mathrm{bp}$ upstream of the initiation codon to $4 \mathrm{bp}$ of the dG-dC tail at the $3^{\prime}$-end after the poly dA-dT tail. The insert of M13-MT27 was excised by cleaving at $X b a \mathrm{I}$ and EcoRI sites of the polylinker and the inserted into pSP64 (22). The resultant plasmid, pSP64-MT27, was cleaved with Eco RI at the distal end of the insert to linearize the plasmid for transcription. M13-MT49 contained the coding sequence of pMT49 together with $34 \mathrm{bp}$ upstream of the ATG and $87 \mathrm{bp}$ of the 3 '-untranslated sequence. M13-MT49 was cleaved with EcoRI and SalI to insert the fragment into pSP65 (22). The resulting plasmid, designated pSP65-MT49, was cleaved with SalI for transcription. The linearized DNA templates $(100 \mu \mathrm{g} / \mathrm{ml})$ were transcribed with SP6 RNA polymerase ( $200 \mathrm{unit} / \mathrm{ml})$ at $37^{\circ} \mathrm{C}$ for $6 \mathrm{~h}$ in the presence of $7 \mathrm{mGpppG}$ as described previoulsy (22).

In vitro translation and analysis of proteins. Hybrid-selected mRNAs and SP6 in vitro transcripts were translated into polypeptides in a rabbit reticulocyte lysate (Amersham) according to the manufacturer's specifications in the presence of ${ }^{35} \mathrm{~S}$-methionine. The translation products were analyzed by SDS polyacrylamide gel electrophoresis (SDS-PAGE) using a $7.5 \%$ acrylamide $-0.2 \%$ bisacrylamide with $4 \%$ stacking gel (16), and by isoelectric focusing (IEF) using a horizontal, flat polyacrylamide gel $(3.8 \%$ acrylamide- $0.2 \%$ bisacrylamide) in $7.5 \mathrm{M}$ urea, LKB ampholines $(1.6 \%$, pH 4-6, and $0.4 \%$, pH 3.5-10) and $2 \%$ NP-40 $(13,25)$. For IEF analysis of in vitro translation products of hybrid-selected RNAs tubulin was concentrated by precipitation with $2 \mathrm{mM}$ vinblastine sulfate as described previously (13). Gels were fixed and prepared for fluorography.

\section{RESULTS}

Cloning of $\beta$-tubulin cDNAs expressed in L5178Y cells. A cDNA library was constructed from the cellular poly (A) ${ }^{+}$RNA of L5178Y cells. Approximately 5,000 cDNA transformants were screened with a nick translated insert excised from the chicken $\beta$-tubulin cDNA clone, pT2. This screening yielded 9 positive clones containing inserts with size around $700 \mathrm{bp}$ and with the same restriction map. The sequencing of these inserts showed that all of the positive clones contained a sequence very similar to the $3^{\prime}$-end portion of the coding region of the chicken $\beta$-tubulin cDNA used as a probe. Since the $\beta$-tubulin mRNAs expressed in L5178Y cells are mainly $1.8 \mathrm{~Kb}$ in size, with a minor band of $2.8 \mathrm{~Kb}$ as revealed by Northern blot analysis of poly (A) ${ }^{+}$RNA of the cells with chicken $\beta$-tubulin cDNA clone probe (data not shown), none of the positive clones contained the complete sequence of $\beta$-tubulin mRNAs. In order to isolate full size $\beta$-tubulin cDNA clones, a new cDNA 
library with inserts of more than $1,500 \mathrm{bp}$ was constructed and screened using a cDNA insert of an isolated clone for mouse $\beta$-tubulin as a probe. In this way, positive clones were isolated from about 20,000 transformants. The inserts of the plasmids from all the positive clones were mapped by digestion with a variety of restriction enzymes. The results showed that 7 clones including pMT27 had the same restriction map. The cDNA clone used as a probe for the second screening contained a partial sequence of this group. The remaining 10 clones had another map which was represented by pMT49.

Nucleotide sequences of cloned cDNAs for $\beta$-tubulin. Sequences of two cDNA clones, pMT27 and pMT49, were analyzed. The insert of pMT27 consists of $69 \mathrm{bp}$ of $5^{\prime}$-untranslated region, $1335 \mathrm{bp}$ of an open reading frame, and $149 \mathrm{bp}$ of the $3^{\prime}$ untranslated region followed by poly (A) tract (data not shown). This sequence is identical with that of a mouse $\beta$-tubulin isotype, $M \beta 3$ (30). The other cDNA clone, pMT49, consists of $179 \mathrm{bp}$ of the $5^{\prime}$-concoding region, $1332 \mathrm{bp}$ of the coding sequence, and $178 \mathrm{bp}$ of the $3^{\prime}$-untranslated region with a poly (A) tract (data not shown). This clone was identified as another mouse $\beta$-tubulin isotype, M $\beta 5$ (30). Their noncoding regions at both $5^{\prime}$ - and $3^{\prime}$-ends have no homology with each other, which allowed us to use the $3^{\prime}$-untranslated sequences of the two cDNA clones as a specific probe for each $\beta$-tubulin (see Materials and Methods).

Identification of polypeptides encoded by two $\beta$-tubulin $c D N A$ s. Isoelectric focusing (IEF) in a flat bed apparatus (Fig. 1B) revealed that there are three major $\beta$-tubulin isoforms in in vitro translation products of poly (A)+ RNA prepared from L5178Y cells (lane 1). Isoelectric points and the relative ratio of the three isoforms did not significantly differ between these in vitro $\beta$-tubulin products and those prepared from cells (Fig. 1B, lane 5). To identify $\beta$-tubulin isoforms encoded by the two cDNA clones, we have analyzed by IEF in vitro translation products of $\beta$-tubulin isotypespecific mRNA selected by hybridization with each of the specific probes described above. The 3'-untranslated regions of the two cDNA inserted into the replicative form of M13 phage were used to select respective mRNAs from total poly (A) ${ }^{+}$RNA of L5178Y cells. The selected RNAs were separately translated into ${ }^{35}$ S-labeled proteins in a reticulocyte lysate. The radio-labeled proteins were analyzed by SDSPAGE and IEF. SDS-PAGE analysis revealed that $\beta$-tubulin with the predicted molecular size $(M r$ ca. 50,000) was synthesized in vitro from the two selected mRNA species (Fig. 1A). We have noted that $\beta$-tubulin synthesized from pMT49(M $\beta 5)$ specific mRNAs moved in SDS-PAGE slightly faster than the counterpart from pMT27(M $\beta 3)$-specific mRNAs. IEF analysis clearly showed that $\beta$-tubulin synthesized from pMT27-specific mRNAs was resolved in a single band which corresponded to $\mathrm{M} \beta \mathrm{Ib}$ although a trace amount of $\mathrm{M} \beta \mathrm{Ia}$ and $\mathrm{M} \beta \mathrm{II}$ was detected when the gel was exposed to X-ray films for a long period (Fig. 1B). This result indicates that pMT27 encodes the $\mathrm{M} \beta \mathrm{Ib}$ isoform. On the other hand, in vitro translation products from pMT49-specfic mRNAs were resolved into two bands, one of which corresponded to $\mathrm{M} \beta \mathrm{Ia}$ and the other to $\mathrm{M} \beta \mathrm{II}$. The molar ratio of the two isoforms ( $\mathrm{M} \beta \mathrm{Ia}: \mathrm{M} \beta \mathrm{II})$ was estimated to be $6: 4$ according to the fluorographic intensity. This ratio did not vary when the stringency of hybrid-selection of mRNA was increased (see Materials and Methods). Both of the two discrete bands on the same IEF gel were proven to be $\beta$-tubulin by examining their mobility in SDS-PAGE after they were cut out from the gel (data not shown).

In vitro translation of SP6 transcripts of $\beta$-tubulin cDNAs. We have examined 


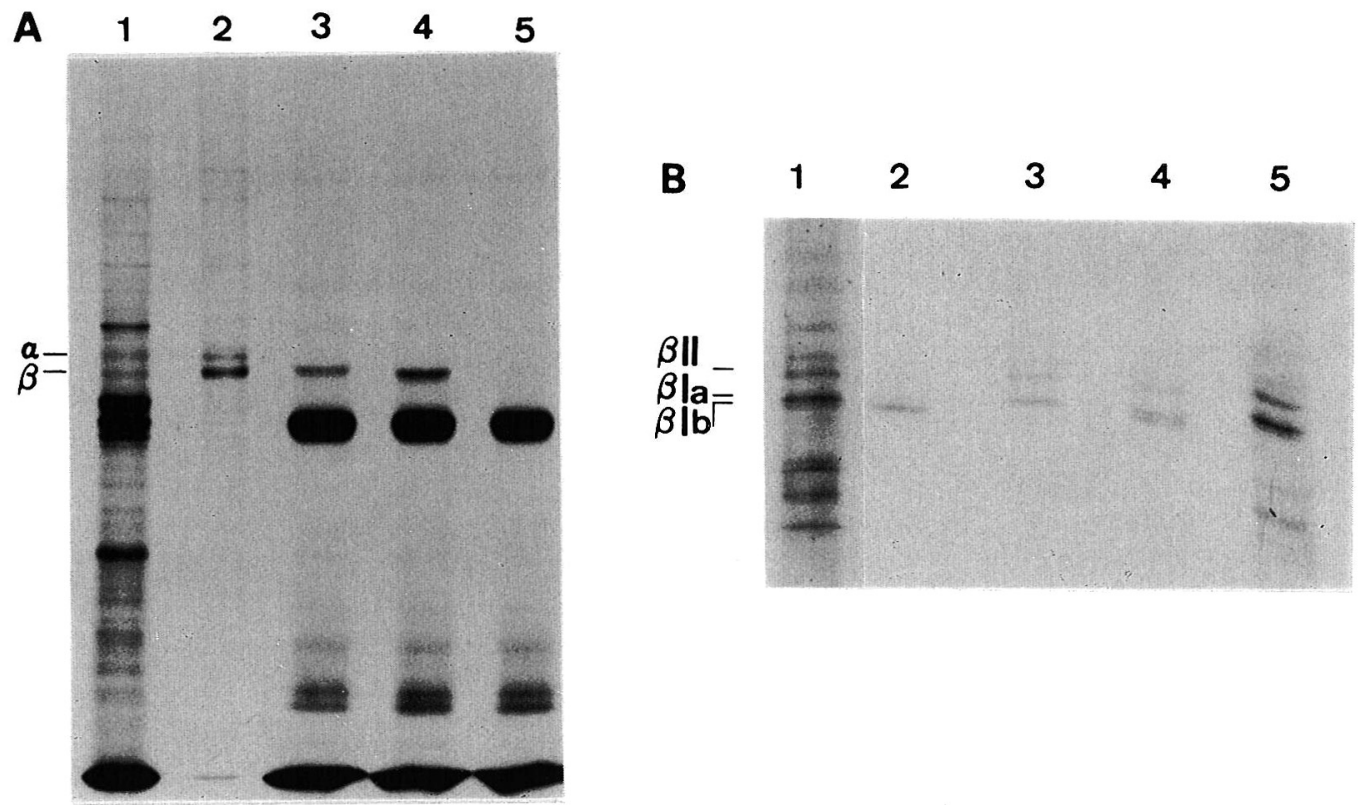

Fig. 1. Analysis of in vitro translation products of mRNAs of L5178Y cells specific for two $\beta$-tubulin cDNAs, pMT27 and pMT49. Poly (A) ${ }^{+}$RNA from L5178Y cells was selected by hybridization with the specific sequences for the two $\beta$-tubulin cDNA clones, which were inserted into the M13-replicative form. Selected RNAs were translated into proteins in a rabbit reticulocyte lysate in the presence of ${ }^{35} \mathrm{~S}$-methionine. Panel A shows SDS-polyacrylamide gel electrophoresis of in vitro translation products of total poly (A) ${ }^{+}$RNA (lane 1), pMT27-specific RNA (lane 3), and pMT49specific RNA (lane 4). $\alpha$ and $\beta$-denote the positions of $\alpha$ and $\beta$-tubulin in the gels, based on the mobilities of tubulin precipitated with vinblastine sulfate (13) from the L5178Y cell lysate (lane 2). Lane 5, control selection in the presence of M13 DNA without an inserted sequence. Panel B shows isoelectric focusing gel electrophoresis of the translation products of tatal poly (A)+ RNA (lane 1), and of vinblastine-precipitates of the translated proteins from pMT27-specific RNA (lane 2), pMT49specific RNA (lane 3), and a mixture of the two samples (lane 4). Isoelectric focusing is from top to bottom (acidic to basic). Positions of the three major isoforms of $\beta$-tubulin are indicated according to those of tubulin prepared from L5178Y cell lysate (lane 5).

whether a single pMT49( $\beta 5)$-specific mRNA species transcribed in vitro from pMT49 DNA is translated into two $\beta$-tubulin isoproteins. By using the SP6 transcription system, RNAs were synthesized in vitro from the two cloned $\beta$-tubulin cDNAs (15, 22). The cDNA inserts of both pMT27 and pMT49 were inserted into pSP64 and pSP65 after removal of their G-C and A-T tails at their 5'-end, giving rise to pSP64MT27 and pSP-MT49, respectively. These two plasmids were linearized at the distal end of the cDNA inserts by cutting with EcoRI and SalI, respectively. Homogeneous SP6 in vitro transcripts were produced from the linearized plasmid DNAs, and translated in vitro in a reticulocyte lysate. We first confirmed by SDS-PAGE that the translation products were homogeneous in size and had the mobility in the gel corresponding to $\beta$-tubulin (Fig. 2A). In accord with the results obtained with $\beta$ tubulin mRNAs selected by hybridization with specific probes (Fig. 1A), $\beta$-tubulin from pSP65-MT49 moved slightly faster than the protein from pSP64-MT27. The protein products synthesized in vitro from the two $\beta$-tubulin cDNAs were resolved 
A

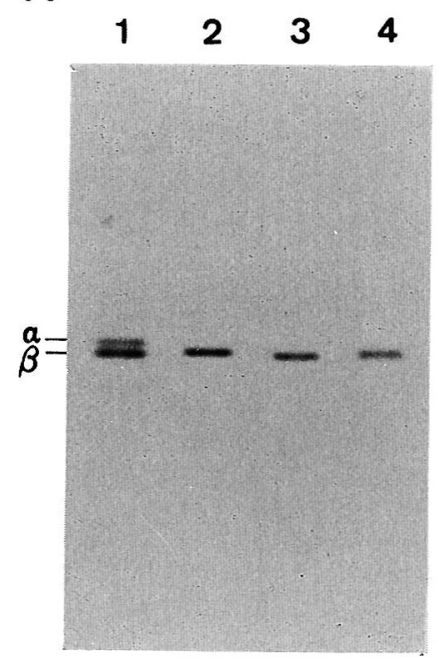

B

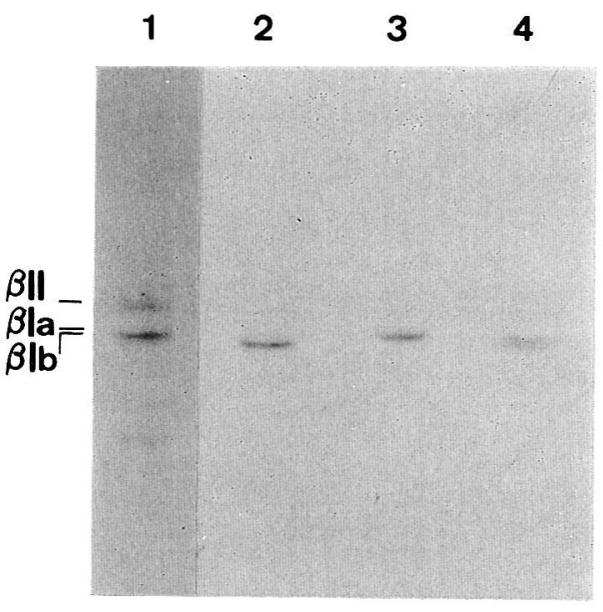

Fig. 2. Translation of SP6 in vitro transcripts of two $\beta$-tubulin cDNAs. The inserts of pMT27 and pMT49 were inserted into pSP64 and pSP65, respectively, as described in MATERIALS AND METHODS, to produce pSP64-MT27 and pSP65-MT49, and transcribed into homogeneous RNAs. Fifty ng of these RNAs were translated into ${ }^{35} \mathrm{~S}$-labeled proteins in $50 \mu \mathrm{l}$ of a rabbit reticulogyte lysate. The reaction mixtures were analyzed by SDS-PAGE (Panel A) and IEF (Panel B). Lane 1, tubulin prepared from the lysate of ${ }^{35} \mathrm{~S}$-labeled L5178Y cells (13). Lane 2, translation product of synthetic RNA from pSP64-MT27. Lane 3, that from pSP65-MT49. Lane 4, the mixture of the above two translation products. " $\alpha$ " and " $\beta$ " indicate $\alpha$-and $\beta$-tubulin, respectively. In Panel B, the three $\beta$-tubulin isoforms are marked. The top of the gels is more acidic and the bottom more basic.

by IEF into unique bands (Fig. 2B). The product from pSP64-MT27 was found to correspond to $\mathrm{M} \beta \mathrm{Ib}$ (lane 2), which is consistent with the hybridization-translation results. On the other hand, the product from pSP65-MT49 corresponded uniquely to $\mathrm{M} \beta$ Ia (lane 3). Thus we have proved that a single $\beta$-tubulin mRNA species is essentially translated into a single $\beta$-tubulin isoform. Based upon these results, we concluded that pMT27 and pMT49 respectively encode the M $\beta$ Ib and M $\beta$ Ia isotypes of $\beta$-tubulin.

\section{DISCUSSION}

We have previously found that L5178Y cells expressed three $\beta$-tubulin isoforms, $\mathrm{M} \beta \mathrm{Ib}, \mathrm{M} \beta \mathrm{Ia}$, and $\mathrm{M} \beta \mathrm{II}$, which can be distinguished in IEF (13). The most acidic isoform, $\mathrm{M} \beta \mathrm{II}$, is not expressed in normal $\mathrm{T}$ cell clones. Furthermore, M $\beta \mathrm{II}$ tubulin has relatively low polymerizability compared to the other two isoforms (13). This would possibly explain the previous finding that L5178Y cells contain a larger amount of unpolymerized tubulin than normal $\mathrm{T}$ cell clones (13). We initially intended to investigate the molecular basis for the lower polymerizability associated with $\mathrm{M} \beta I$. Before conducting this investigation, we attempted to determine the origins of three different $\beta$-tubulin isoforms expressed in L5178Y cells. Multiple tubulin isoforms occur as products of a small multigene family (28), by post-translational modifications such as phosphorylation (10), or possibly by post-transcriptional processing. This 
study was originally designed to determine which is the case(s) in the generation of the three $\beta$-tubulin isoforms expressed in L5178Y cells.

For this purpose, we isolated seventeen full length $\beta$-tubulin cDNA clones prepared from the poly (A) ${ }^{+}$RNA of L5178Y cells. These cDNA clones were classified into two mouse $\beta$-tubulin isotypic cDNA clones, pMT27 and pMT49, according to the results of restriction mapping. Both pMT27 and pMT49 contained full length $\beta$-tubulin cDNAs including the 5 '-noncoding sequences. To identify $\beta$-tubulin isoforms encoded by the two cDNA clones among the three isoforms expressed in L5178Y cells, we conducted two experiments: (a) analysis of in vitro translation products of hybrid-selected mRNAs with $\beta$-tubulin isotype-specific probes; (b) similar analysis of products of SP6 in vitro transcripts from the two cDNAs (28). Use of the above in vitro translation system seems to be sound because the three isoforms were produced in this system by using total poly (A)+ RNAs of L5178Y cells as templates. These two experiments gave consistent results regarding the isoform encoded by pMT27; that is, pMT27 encodes M $\beta$ Ib. In contrast, two polypeptides corresponding to $\mathrm{M} \beta \mathrm{Ia}$ and $\mathrm{M} \beta \mathrm{II}$ were produced in vitro from mRNA selected with the specific sequence for pMT49. This result may be explained in two ways. First, two mRNA species with common or very similar sequences of the $3^{\prime}$-untranslated regions present in L5178Y cells are templates for $\mathrm{M} \beta \mathrm{Ia}$ and $\mathrm{M} \beta \mathrm{II}$. Alternatively, a single polypeptide species produced from mRNA corresponding to pMT49 was post-translationally modified, resulting in two $\beta$-tubulin proteins separable by IEF. If the latter is the case, the in vitro transcript of pMT49 could be translated into those two proteins in the same in vitro translation system. As shown here, that did not turn out to be the case, however. We would, therefore, expect that mRNA to be translated into M $\beta I I$, which has a similar 3'-untranslated sequence to that of pMT49, might be present in L5178Y cells. We had not previously expected such a case since intraspecies-conservation of the $3^{\prime}$-noncoding region had not been observed so far among distinct $\beta$-tubulin isotypes. Southern blot analysis of the whole genomic DNA of L5178Y cells with the specific probe for pMT49 gave a single band (unpublished observation), suggesting the possibility that $\mathrm{M} \beta \mathrm{Ia}$ and $\mathrm{M} \beta \mathrm{II}$ might be separately encoded by the same gene on paired chromosomes of the L5178Y lymphoma strain. However, this and other possibilities too remain to be elucidated.

Acknowledgements. We thank Drs. D.W. Cleaveland and M. Yamamoto for supplying chicken $\beta$-tubulin cDNA clone. We thank also the other members of Department of Biochemistry, the Cancer Institute for their technical advice and Dr. S. Koyasu for his help in RNA preparation.

This work was supported in part by Grants-in-Aid from the Ministry of Education, Science and Culture of Japan, by Special Coordination Funds for Promoting Science and Technology Agency of Japan, and by the Toray Science Foundation.

\section{REFERENCES}

1. Aviv, H. and P. Leder. Purification of biologically active globin messenger RNA by chromatography on oligothymidylic acid-cellulose. Proc. Natl. Acad. Sci. U.S.A. 69, 14081412, 1972

2. Bond, J.F., G.S. Robinson and S.R. FARMER. Differential expression of two neural cellspecific $\beta$-tubulin mRNAs during rat brain development. Mol. Cell. Biol. 4, 1313-1319, 1984

3. Brown, B.A., R.A. Nixon and C.A. Marotta. Posttranslational processing of $\alpha$-tubulin during axoplasmic transport in CNS axons. J. Cell Biol. 94, 159-164, 1982 
4. Cleveland, D.W. The multitubulin hypothesis revisited: What have we learned? J. Cell Biol. 104, 81-383, 1987

5. Denoulet, P., C. Jeantet and F. Gros. Tubulin microheterogeneity during mouse liver development. Biochem. Biophys. Res. Commun. 105, 806-813, 1982

6. Dustin, P. Microtubules, 2nd Ed., Springer-Verlag, Berlin, 1983

7. Favaloro, J., R. Treisman and R. Kamen. Transcription maps of polyoma virus-specific RNA: Analysis by two-dimensional nuclease Sl gel mapping. Meth. Enzymol. 65, 718-749, 1980

8. Field, D.J., R.A. Collins and J.C. Lee. Heterogeneity of vertebrate brain tubulins. Proc. Natl. Acad. Sci. U.S.A. 81, 4041-4045, 1984

9. Fulton, C. and P.A. Simpson. Selective synthesis and utilization of flagellar tubulin. The multi-tubulin hypothesis. In Cell Motility, ed. Goldman, R., T. Pollard and J. Rosenbaum., Cold Spring Harbor Laboratory, New York, pp. 987-1005, 1976

10. GaRD, D.L. and M.W. KirSChNer. A polymer-dependent increase in phosphorylation of $\beta$-tubulin accompanies differentiation of a mouse neuroblastoma cell line. J. Cell Biol. 100, 764-774, 1985

11. GonZes, I. and K.J. SweAdNer. Multiple tubulin forms are expressed by a single neurone. Nature 294, 477-480, 1981

12. Grunstein, M. and D.S. Hogness. Colony hybridization: A method for isolation of cloned DNAs that contain a specific gene. Proc. Natl. Acad. Sci. U.S.A. 72, 3961-3965, 1975

13. Harada, F., F. Matsuzaki and I. Yahara. Occurrence of two $\beta$-tubulin isoforms with different polymerizing abilities in L5178Y cells. Exp. Cell Res. 166, 265-278, 1986

14. Havercroft, J.C. and D.W. Cleveland. Programmed expression of $\beta$-tubulin genes during development and differentiation of the chicken. J. Cell Biol. 99, 1927-1935, 1984

15. KRIEG, P.A. and D.A. Melton. Functional messenger RNAs are produced by SP6 in vitro transcription of cloned cDNAs. Nuc. Acid Res. 12, 7057-7070, 1984

16. Laemmli, U.K. Cleavage of structural proteins during the assembly of the head of bacteriophage T4. Nature 227, 680-685, 1970

17. Land, H., M. Grez, H. Hauser, W. Lindenmaier and G. Schutz. 5'-Terminal sequences of eucaryotic mRNA can be cloned with high efficiency. Nuc. Acid Res. 9, 2251-2266, 1981

18. Lewis, S.A., M.E. Gilmartin, J.L. Hall and N.J. Cowan. Three expressed sequences within the human $\beta$-tubulin multigene family each define a distinct isotype. J. Mol. Biol. 182, $11-20,1985$

19. Lewis, S.A., M.G-S. LeE and N.J. Cowan. Five mouse tubulin isotypes and their regulated expression during development. J. Cell Biol. 101, 852-861, 1985

20. Maniatis, T., Fritsch E.F. and J. Sambrook. In Molecular Cloning, Cold Spring Harbor Laboratory, New York, pp. 229-246, 1982

21. MaXam, A.M. and W. Gilbert. Sequencing end-labeled DNA with base-specific chemical cleavages. Meth. Enzymol. 65, 499-560, 1980

22. Melton, D.A., P.A. Krieg, M.R. Rebagliati, T. Maniatis, K. Zinn and M.R. Green. Efficient in vitro synthesis of biologically active RNA and RNA hybridization probes from plasmids containing a bacteriophage SP6 promoter. Nuc. Acid Res. 12, 7035-7056

23. NATZLE, J.E. and B.J. MCCARTHY. Regulation of Drosophila $\alpha$ - and $\beta$-tubulin genes during development. Dev. Biol. 104, 187-198, 1984

24. Norrander, J., T. Kemper and J. Messing. Construction of improved M13 vectors using oligonucleotide-directed mutagenesis. Gene 26, 101-106, 1983

25. O'Farrell, P.H. High resolution two-dimensional electrophoresis of proteins. J. Biol. Chem. 250, 4007-4021, 1975

26. Parnes, J.R., B. Velan, A. Felsenfeld, L. Ramanathan, U. Ferrini, E. Appella and J.G. Seidman. Mouse $\beta 2$-microglobulin cDNA clones: A screening to rare mRNAs. Proc. Natl. Acad. Sci. U.S.A. 78, 2253-2257, 1981

27. Sanger, F., A.R. Coulson, B.G. Barrel, A.J.H. Smith and R.A. Roe. Cloning in singlestranded bacteriophage as an aid to rapid DNA sequencing. J. Mol. Biol. 143, 161-178, 1980

28. Sullivan, K.F., J.C. Havercroft and D.W. Cleveland. Primary structure and expression 
of a vertebrate $\beta$-tubulin gene family. In Molecular Biology of the Cytoskeleton, ed. Borisy, G.G., D.W. Cleveland and D.B. Murphy, Cold Spring Harbor Laboratory, New York, pp. 321-332, 1984

29. Valenzuela, P., M. Quiroga, J. Zaldiver, W.J. Rutter, M.W. Kirschner and D.W. Cleveland. Nucleotide and corresponding amino acid sequences encoded by $\alpha$ and $\beta$-tubulin mRNAs. Nature 289, 650-655, 1981

30. Wang, D., A. Villasante, S.A. Lewis and N.J. Cowan. The mammalian $\beta$-tubulin repertoire: hematopoietic expression of a novel, heterologous $\beta$-tubulin isotype. J. Cell Biol. 103, 19031910, 1986

(Received for publication, April 17, 1987) 